\section{BMJ Open Respiratory Research}

\title{
British Thoracic Society Quality Standards for outpatient management of pulmonary embolism
}

Robin Condliffe, ${ }^{1}$ Paul Albert, ${ }^{2}$ Raza Alikhan, ${ }^{3}$ Emma Gee, ${ }^{4}$ Daniel Horner, ${ }^{5}$ Laura Hunter, ${ }^{6}$ Phillip Jacobs, ${ }^{7}$ Rachel Limbrey, ${ }^{8}$ Michael Newnham, ${ }^{9}$ Wendy Preston, ${ }^{10}$ Sheena Patel, ${ }^{11}$ Laura-Jane Smith, ${ }^{12}$ Jay Suntharalingam ${ }^{13}$

To cite: Condliffe $R$, Albert $P$, Alikhan R, et al. British Thoracic Society Quality Standards for outpatient management of pulmonary embolism. BMJ Open Resp Res Published Online First: [please include Day Month Year]. doi:10.1136/ bmjresp-2020-000636

- Additional material is published online only. To view, please visit the journal online (http://dx.doi.org/10. 1136/bmjresp-2020-000636).

Received 16 May 2020 Revised 27 May 2020 Accepted 28 May 2020
Check for updates

(C) Author(s) (or their employer(s)) 2020. Re-use permitted under CC BY-NC. No commercial re-use. See rights and permissions. Published by BMJ.

For numbered affiliations see end of article.

Correspondence to Dr Robin Condliffe; robin.condliffe@sth.nhs.uk

\section{ABSTRACT}

Introduction The purpose of the quality standards document is to provide healthcare professionals, commissioners, service providers and patients with a guide to standards of care that should be met for outpatient management of pulmonary embolism in the UK, together with measurable markers of good practice. Quality statements are based on the British Thoracic Society (BTS) Guideline for the Initial Outpatient Management of Pulmonary Embolism.

Methods Development of BTS Quality Standards follows the BTS process of quality standard production based on the National Institute for Health and Care Excellence process manual for the development of quality standards.

Results Six quality statements have been developed, each describing a standard of care for the outpatient management of pulmonary embolism in the UK, together with measurable markers of good practice. Discussion BTS Quality Standards for Outpatient Management of Pulmonary Embolism form a key part of the range of supporting materials that the society produces to assist in the dissemination and implementation of a guideline's recommendations.

\section{INTRODUCTION}

The British Thoracic Society (BTS) has been at the forefront of the production of guidelines for best clinical practice in respiratory medicine since the Society was established over 30 years ago. Guideline production methodology has evolved considerably in recent years, and a manual setting out the detailed policy for the production of BTS Guidelines is reviewed annually by the BTS Standards of Care Committee (SOCC). BTS Guidelines received National Institute for Health and Care Excellence (NICE) accreditation in 2011. The production of quality standards based on each BTS Guideline is a key part of the range of supporting materials that the Society produces to assist in the dissemination and implementation of a guideline's recommendations. The purpose of the quality standards document is to provide healthcare professionals, commissioners, service providers and patients with a guide to standards of care that should be met for the outpatient management of acute pulmonary embolism (PE) in the $\mathrm{UK}$, together with measurable markers of good practice. BTS Quality Standards are intended for:

- Healthcare professionals to allow decisions to be made about care based on the latest evidence and best practice.

- People with respiratory disease and their families and carers to enable understanding of what services they should expect from their health and social care provider.

- Service providers to be able to quickly and easily examine the clinical performance of their organisation and assess the standards of care they provide.

- Commissioners (or equivalent in Scotland and Northern Ireland) so that they can be confident that the services they are purchasing are high quality and cost-effective.

NICE Quality Standards were used as a model for the development of BTS Quality Standards, and the development of these quality standards is based on the NICE Quality Standards Process Guide. ${ }^{1}$ This document contains quality standards for the outpatient management of PE. This document was approved by the BTS SOCC in March 2020.

A quality standard is a set of specific, concise statements that:

- act as markers of high-quality, costeffective patient care across a pathway or clinical area, covering treatment or prevention;

- are derived from the best available evidence. 
The development of these quality standards was stimulated, in part, by the National Confidential Enquiry into Patient Outcome and Death: Know the Score 2019 (https://www.ncepod.org.uk/2019pe.html), which was published in October 2019.

The BTS Quality Standards include evidence and recommendations summarised in the BTS Guideline for the Initial Outpatient Management of Pulmonary Embolism (PE), which was published in 2018. ${ }^{2}$

Each quality standard includes the following:

- A quality statement, which describes a key marker of high-quality, cost-effective care for this condition.

- Quality measures, which aim to improve the structure, process and outcomes of healthcare.

The quality measures are not intended to be new sets of targets or mandatory indicators for performance management that need to be collected. The quality measures are specified in the form of a numerator and a denominator, which define a proportion or ratio (numerator/denominator). It is assumed that the numerator is a subset of the denominator population. The suggested numerator and denominator are provided to allow healthcare professionals and service providers to examine their clinical performance in relation to each quality standard. It is recognised that no national quality indicators will be available for this condition, and institutions will need to agree locally what information is required for the denominator to be used in each case and what the expected level of achievement should be, given local circumstances. A brief description about the quality standard in relation to each audience is given.

The main source reference for these Quality Standards is the BTS Guideline for the initial outpatient management of PE, 2018. ${ }^{2}$ There is no specific order of priority associated with the list of quality statements.

\section{METHOD OF WORKING}

A Quality Standards Working Group was convened in November 2018 and met in January 2019. Table 1 shows the membership of the group. Members of the Quality Standards Group submitted Declaration of Interest forms in line with the BTS policy, and copies of forms are available on request from BTS Head Office.

The draft document was considered in detail by the BTS SOCC initially in June 2019 and the BTS Quality Improvement Committee in June 2019. The document was made available on the BTS website for public consultation for the period from September to October 2019. Following further revision, the document was submitted for approval to the BTS SOCC in March 2020. The Quality Standards document will be reviewed in 2025, or following the publication of a revised guideline whichever is the sooner. Lay input to the development of the BTS Quality Standards was provided through the lay/patient members of the BTS Standards of Care Committee, which reviewed the document and provided final sign off.

\begin{tabular}{|c|c|}
\hline Dr Robin Condliffe & Chair, Consultant Respiratory Physician, Royal Hallamshire Hospital, Sheffield \\
\hline Dr Paul Albert & Consultant Respiratory Physician, Aintree Hospital, Liverpool \\
\hline Dr Raza Alikhan & $\begin{array}{l}\text { Consultant Haematologist, University Hospital, Wales, Cardiff representing the British Society for } \\
\text { Haematology }\end{array}$ \\
\hline Emma Gee & Nurse Consultant, Thrombosis and Coagulation, King's College Hospital, London \\
\hline Dr Daniel Horner & $\begin{array}{l}\text { Consultant Physician, Emergency and Intensive Care Medicine, Salford Royal NHS Foundation } \\
\text { Trust, representing the Royal College of Emergency Medicine }\end{array}$ \\
\hline Dr Laura Hunter & $\begin{array}{l}\text { Consultant Physician Emergency Medicine, London, representing the Royal College of Emergency } \\
\text { Medicine }\end{array}$ \\
\hline Dr Phil Jacobs & $\begin{array}{l}\text { Consultant Acute Physician, Royal Free London NHS Foundation Trust, representing the Society for } \\
\text { Acute Medicine }\end{array}$ \\
\hline Dr Rachel Limbrey & Consultant Respiratory Physician, University Hospital Southampton \\
\hline Dr Michael Newnham & Respiratory Specialty Trainee, University of Birmingham \\
\hline Wendy Preston & Head of Nursing Practice, Royal College of Nursing, Consultant Nurse, George Eliot Hospital \\
\hline Sheena Patel & $\begin{array}{l}\text { Lead Pharmacist, Anticoagulation and Medication Safety/Clinical Governance, Chelsea and } \\
\text { Westminster Hospital, London }\end{array}$ \\
\hline Dr Laura-Jane Smith & Respiratory Specialty Trainee, London \\
\hline Dr Jay Suntharalingam & Consultant Respiratory Physician, Royal United Hospital, Bath \\
\hline
\end{tabular}




\section{LIST OF QUALITY STATEMENTS}

1: CT pulmonary angiography (CTPA) should be performed within 24 hours of presentation in patients who are managed via an outpatient pathway and do not have contraindications for contrast imaging.

2: All patients with confirmed acute PE or on an outpatient pathway for suspected acute PE should have their clinical risk assessed including the use of a validated risk score (PE severity index (PESI), simplified PESI (s-PESI), Hestia).

3: Outpatient management should be offered to all patients with suspected or confirmed acute PE who satisfy clinical risk and exclusion criteria.

4: All patients managed via an outpatient PE pathway should be reviewed by a senior clinical decision-maker prior to going home.

5: All patients managed via an outpatient PE pathway should receive verbal and written information containing details of potential complications of the disease process, its treatment and a point of contact.

6: Patients undergoing outpatient management following diagnosis of an acute PE should have an initial review within 7 days of discharge. Subsequent follow-up by a senior clinician with a special interest in PE should take place within a formal pathway.

\section{Quality statement 1}

Rationale

\section{Quality measure}

Description of what the quality statement means for each audience

\section{Relevant existing indicators/data sources}

\section{Source references}

CTPA should be performed within 24 hours of presentation in patients who are managed via an outpatient pathway and do not have contraindications for contrast imaging.

- Delays in radiological investigation of suspected PE may result in both unnecessary repeat doses of anticoagulation and delay in diagnosing alternative pathology in patients without an acute PE. Performing and reporting CTPA within 24 hours of hospital presentation will reduce the risk of these issues occurring.

\section{Structure:}

Evidence of local arrangements to ensure that all suitable patients managed on an outpatient pathway* can:

- Undergo a CTPA within 24 hours of presentation to hospital.

- Have the results of that investigation reported (including comments on the presence or absence of right ventricular dilatation on CTPA) within 24 hours of presentation.

*Patients in whom PE has been excluded by a non-high clinical likelihood score and negative D-

Dimer are not considered, in these Quality Standards, to be on an outpatient pathway.

Process:

- The proportion of suitable patients on an outpatient pathway who undergo CTPA within 24 hours of presentation to hospital.

- The proportion of patients whose CTPA images are reported within 24 hours of presentation.

Numerator 1: The number of suitable patients on an outpatient pathway who undergo CTPA within 24 hours of presentation.

Denominator 1: The total number of suitable patients on an outpatient pathway for the management of PE.

Numerator 2: The number of CTPA scans performed in patients on an outpatient pathway, which are reported (including comments on the presence or absence of right ventricular dilatation on CTPA) within 24 hours of presentation.

Denominator 2: The total number of CTPA scans performed in patients on an outpatient pathway for the management of PE.

\section{Service providers:}

- Ensure systems are in place to enable extended access to CTPA and imaging and reporting 7 days a week.

Healthcare professionals:

- Ensure that suitable patients are referred for CTPA promptly and that images are reported and communicated to clinical teams efficiently.

Commissioners:

- Ensure that sufficient facilities, staff and equipment are available to ensure that suitable patients undergo CTPA within 24 hours of presentation.

People on an outpatient pathway:

- People who are on an outpatient pathway should undergo CTPA, where there are no contraindications for contrast imaging, within 24 hours of presentation, and the reports of those investigations should be made available to the responsible hospital clinical team.

- BTS Guideline for the Initial Outpatient Management of Pulmonary Embolism (2018). ${ }^{2}$

> NICE Clinical Guidelines 144 (2015). ${ }^{3}$

- NICE Quality Standards 29 (2013). ${ }^{4}$

- Royal College of Physicians of Edinburgh and Society of Acute Medicine; Standardsfor Ambulatory Emergency Care (2019) ${ }^{5}$

- BTS Guideline for the Initial Outpatient Management of Pulmonary Embolism (2018). ${ }^{2}$ 
Definitions
Outpatient pathway: a formalised pathway whereby patients with confirmed PE are discharged home on the same day as diagnosis while patients with suspected PE may be discharged home following initial assessment to subsequently return to hospital for definitive investigation.

Suitable patients: patients who do not have contraindications to CTPA (eg, previous contrast reaction or significantly impaired renal function).
Quality statement 2

Rationale

Quality measure

\section{Description of what the quality statement means for each audience}

\section{Relevant existing indicators/data sources}

\section{Source references}

\section{Definitions}

\section{All patients with confirmed acute PE or on an outpatient pathway for suspected acute PE should have their clinical risk assessed including the use of a validated risk score (PESI, s- PESI, Hestia).}

- Early risk stratification of patients with suspected or confirmed acute PE identifies those at higher and lower risk of death. The use of a risk stratification tool, such as PESI and s-PESI (online supplementary appendices 1-2), therefore enables patients to be optimally managed in the most appropriate setting within hospital or in their own home.

- Clinical exclusion tools, such as the Hestia criteria (online supplementary appendix 3), have also been developed to identify people who are not suitable for outpatient management.

- It is recommended that all patients with confirmed acute PE or on an outpatient pathway for suspected acute PE undergo risk stratification using one of these tools before a decision is made to manage them in an outpatient setting.

\section{Structure:}

- Evidence that all patients with confirmed acute PE or on an outpatient pathway for suspected acute PE have been risk-stratified using a validated clinical risk score (eg, PESI, s-PESI, Hestia).

\section{Process:}

- The proportion of patients presenting to hospital with a new presentation of confirmed acute PE or on an outpatient pathway for suspected acute PE who have been risk stratified using a validated clinical risk score.

Numerator: The number of patients presenting to hospital with a new presentation of confirmed acute PE or on an outpatient pathway for suspected acute PE who have been risk stratified using a validated clinical risk score.

Denominator: The total number of patients presenting to hospital with a new presentation of confirmed acute PE or on an outpatient pathway for suspected acute PE.

\section{Service providers:}

- Should ensure that clinicians who assess patients with confirmed acute PE or patients on an outpatient pathway for suspected acute PE are appropriately trained and have sufficient time to carry out clinical risk assessments in these patients.

- Should ensure that local guidelines and standard operating procedures are in place which provide sufficient guidance on carrying out risk assessments.

Healthcare professionals:

- Should ensure that they are adequately trained to carry out risk assessments in patients with confirmed acute PE or patients on an outpatient pathway for suspected acute PE, and that they use the outcomes from risk assessments to guide management.

Commissioners:

- Should ensure that they commission services that have local guidelines and standard operating procedures in place regarding clinical risk assessments and that are adequately resourced to carry these out.

People who present with confirmed acute PE or patients on an outpatient pathway for suspected acute PE

- Should expect to undergo a clinical risk assessment to help determine the most appropriate place for them to receive their initial care.

- BTS Guideline for the Initial Outpatient Management of Pulmonary Embolism (2018). ${ }^{2}$

> NICE Clinical Guidelines $144(2015){ }^{3}$

- NICE Quality Standards 29 (2016). ${ }^{4}$

- Royal College of Physicians of Edinburgh and Society of Acute Medicine; Standards for Ambulatory Emergency Care (2019) ${ }^{5}$

- BTS Guideline for the Initial Outpatient Management of Pulmonary Embolism (2018). ${ }^{2}$

- Derivation and validation of a prognostic model for pulmonary embolism (2005). ${ }^{6}$

- Simplification of the pulmonary embolism severity index for prognostication in patients with acute symptomatic pulmonary embolism (2010). ${ }^{7}$

> Outpatient treatment in patients with acute pulmonary embolism: the Hestia Study (2011). ${ }^{8}$

Suspected PE: clinical suspicion of PE (history, symptoms, signs, PE likelihood score and initial investigations including $D$-Dimer) without a radiological diagnosis. 
Quality statement 3

Rationale

Quality measure

Description of what the quality statement means for each audience

\section{Relevant existing indicators/data sources}

Source references

Definitions
Outpatient management should be offered to all patients with suspected or confirmed acute PE who satisfy clinical risk and exclusion criteria

Outpatient management of PE is safe in properly selected low-risk patients, with non-inferior rates of recurrent venous thromboembolism, major bleeding, PE-related death and with equivalent patient satisfaction compared with inpatient care. ${ }^{19-13}$ Outpatient management of PE results in reduced length of stay, which may translate to healthcare-related cost savings. ${ }^{10121415}$ Outpatient pathways are, however, currently under-utilised. ${ }^{16}$

Structure:

- Evidence that local arrangements are in place to ensure that eligible patients with suspected or confirmed PE are offered outpatient care.

- Eligibility for outpatient care should be assessed by clinical risk stratification and assessment of exclusion criteria.

Process:

- The proportion of eligible patients presenting to hospital with suspected or confirmed PE who are offered outpatient management.

Numerator: The number of patients with suspected or confirmed acute PE who satisfy clinical risk and exclusion criteria who are offered outpatient management.

Denominator: The number of patients with suspected or confirmed PE who satisfy clinical risk and exclusion criteria for outpatient management.

\section{Service providers:}

- Should ensure systems and staffing are in place for people with suspected or confirmed PE to be offered outpatient PE management if they fulfil eligibility criteria.

Healthcare professionals:

- Should ensure people presenting to hospital with suspected or confirmed PE are offered outpatient PE management via a dedicated care pathway if they fulfil eligibility criteria. They should provide adequate information to allow patients to participate in decisions regarding outpatient management.

\section{Commissioners:}

- Should ensure that services are commissioned with sufficient capacity and resources to provide outpatient PE management to those patients fulfilling eligibility criteria.

\section{People who have suspected or confirmed PE:}

- Should be offered outpatient PE management if they fulfil eligibility criteria. To enable them to make an informed decision regarding outpatient management, they should have the opportunity for a discussion regarding the risks and benefits of outpatient care with a healthcare professional who possesses the necessary knowledge and skills.

- BTS Guideline for the Initial Outpatient Management of Pulmonary Embolism (2018). ${ }^{2}$

- BTS Guideline for the Initial Outpatient Management of Pulmonary Embolism (2018). ${ }^{2}$

- Home treatment in pulmonary embolism (2010). ${ }^{9}$

- Outpatient vs inpatient treatment for patients with acute pulmonary embolism: an international, open-label, randomised, non-inferiority trial (2011). ${ }^{10}$

- Outpatient treatment of symptomatic pulmonary embolism: a systematic review and metaanalysis $(2013)^{11}$

- Early discharge of patients with pulmonary embolism: a two-phase observational study $(2007)^{12}$

- Out of hospital treatment of acute pulmonary embolism in patients with a NT-proBNP level (2010)..$^{13}$

- Investigating and managing suspected pulmonary embolism in an outpatient setting: the Leicester experience $(2014)^{14}$

- Home treatment of patients with small-sized to medium-sized acute pulmonary embolism (2014). ${ }^{15}$

- Rate and duration of hospitalisation for deep vein thrombosis and pulmonary embolism in realworld clinical practice (2015). ${ }^{16}$

Outpatient management: patients with confirmed PE are discharged home on the same day as diagnosis while patients with suspected PE may be discharged home following initial assessment to subsequently return to hospital for definitive investigation.

Eligibility criteria: either (a) clinical risk score (eg, PESI or s-PESI) plus clinical exclusion criteria or

(b) clinical exclusion score (eg, Hestia) as described in the BTS Guideline for the Initial Outpatient Management of Pulmonary Embolism (2018). ${ }^{2}$ 
Quality statement 4

Rationale

Quality measure

Description of what the quality statement means for each audience

Relevant existing indicators/data sources

Source references

Definitions

Other information
All patients managed via an outpatient PE pathway should be reviewed by a senior clinical decision-maker prior to going home.

Patients with either suspected or confirmed acute PE require review by a senior clinical decisionmaker prior to discharge home to ensure that they are suitable for outpatient management and that other potential causes for symptoms have been excluded.

\section{Structure:}

- Evidence of local arrangements to ensure that patients managed via an outpatient pathway are reviewed by a senior clinical decision-maker with access to an on-call consultant.

\section{Process:}

- The proportion of patients with suspected or diagnosed acute PE managed via an outpatient pathway who are reviewed by a senior clinical decision-maker prior to going home.

Numerator 1:The number of patients with suspected or confirmed acute PE who are reviewed by a senior clinical decision-maker prior to going home via an outpatient pathway.

Denominator 1:The number of patients with suspected or confirmed acute PE managed via an outpatient pathway.

Service providers:

- Should ensure there are adequate systems, staffing and support services in place to provide outpatient management pathways for eligible patients with suspected or confirmed acute PE.

Healthcare professionals:

- Should ensure that all patients with suspected or confirmed acute PE who are eligible for management within an outpatient pathway are reviewed by a senior clinical decision-maker prior to going home.

Commissioners:

- Should ensure that they commission services with sufficient capacity, staff and consultant oversight in order to deliver safe outpatient pathways for the management of suspected and confirmed acute PE.

People who are managed on an outpatient pathway for suspected or confirmed PE:

- Should receive a review by an appropriate senior clinical decision-maker prior to going home.

- BTS Guideline for the Initial Outpatient Management of Pulmonary Embolism (2018). ${ }^{2}$

- Royal College of Physicians of Edinburgh and Society of Acute Medicine. Standards for Ambulatory Emergency Care (2019). ${ }^{5}$

- BTS Guideline for the Initial Outpatient Management of Pulmonary Embolism (2018). ${ }^{2}$

Senior clinical decision maker: a consultant physician, or if no consultant is available, a senior trainee (ST3 or above, ST4 in the case of emergency medicine), a staff grade or similar substantive career grade doctor or an advanced nurse practitioner or clinical nurse specialist with sufficient experience and training to be designated to undertake this role under an appropriately trained supervisor.

Example of patient information leaflets-see Appendix 4: King's College Hospital NHS Foundation Trust, Royal Free London NHS Foundation Trust and Sheffield Teaching Hospitals NHS Foundation Trust.
Quality statement 5

Rationale

\section{Quality measure}

All patients managed via an outpatient PE pathway should receive verbal and written information containing details of potential complications of the disease process, its treatment and a point of contact.

In view of the nature of outpatient management, patients should be supplied with verbal and written information containing information regarding the diagnosis of PE, its treatment and symptoms suggestive of complications, together with a point of contact.

\section{Structure:}

- Evidence of local arrangements to ensure that patients managed via an outpatient pathway receive adequate verbal and written information.

Process:

- The proportion of patients with suspected or diagnosed acute PE managed via an outpatient pathway who receive verbal and written information prior to going home.

Numerator 1: The number of patients with suspected or confirmed acute PE managed via an outpatient pathway in whom there is clear evidence of the provision of verbal and written information including potential complications of the disease, treatment and a focal contact point.

Denominator 1: The number of patients with suspected or confirmed acute PE managed via an outpatient pathway. 
Description of what the quality statement audience

\section{Relevant existing indicators/data sources}

Source references Definitions means for each

\section{Service providers:}

- Should ensure that written information regarding the condition, potential complications, symptoms of recurrence, treatment and contact information is available.

Healthcare professionals:

- Should ensure that all patients with suspected or confirmed acute PE who are eligible for management within an outpatient pathway are provided with specific verbal and written information prior to going home.

Commissioners:

- Should ensure that they commission services which can demonstrate provision of adequate written information.

People who are managed on an outpatient pathway for suspected or confirmed PE:

- Should receive verbal and written information regarding the disease, complications of the disease process and treatments and be provided with a clear point of contact for clinical queries and concerns.

- BTS Guideline for the Initial Outpatient Management of Pulmonary Embolism (2018). ${ }^{2}$

- Royal College of Physicians of Edinburgh and Society of Acute Medicine. Standards for Ambulatory Emergency Care (2019). ${ }^{5}$

- BTS Guideline for the Initial Outpatient Management of Pulmonary Embolism (2018). ${ }^{2}$

Other information: Example of patient information leaflets - see online supplementary appendix 4: King's College Hospital NHS Foundation Trust, Royal Free London NHS Foundation Trust and Sheffield Teaching Hospitals NHS Foundation Trust.

Quality statement 6

Patients undergoing outpatient management following diagnosis of an acute PE should have an initial review within 7 days of discharge. Subsequent follow-up by a senior clinician with a special interest in PE should take place within a formal pathway.

Rationale

Patients managed via an outpatient pathway require assessment within the first 7 days to enable:

- Assessment of ongoing symptoms.

- Review of concordance with treatment as adequate early anticoagulation is imperative to minimise risk of recurrence.

- Assessment of side effects, including bleeding complications.

- A check that limited screening for underlying malignancy has been completed in all patients without known cancer, with referral for more extensive screening in selected cases, dependent on results of the initial investigations.

- Further discussion regarding PE and expected recovery process.

Subsequent follow-up by a senior clinician with a special interest in PE after 3-6 months enables an individualised plan for ongoing anticoagulation to be made, based on the presence or absence of provoking factors and risk factors for bleeding. It also provides an opportunity to discuss other factors related to PE. Furthermore, it allows assessment of any ongoing symptoms of breathlessness, with subsequent investigations for the presence of chronic thromboembolic pulmonary hypertension in selected cases. This follow-up will most often be provided by a doctor, but in some instances may be a specialist nurse or an advanced practitioner who fulfils the Royal College of Nursing standards (https://www.rcn.org.uk/professional-development/advanced-practicestandards).

\section{Quality measure}

\section{Structure:}

- Evidence of local arrangements and written clinical protocols and pathways that ensure patients with a new diagnosis of PE are offered robust follow-up.

Process:

- The proportion of patients with confirmed acute PE who are reviewed within 7 days and subsequently, within an outpatient pathway.

Numerator 1: The number of patients treated via an outpatient pathway with a new diagnosis of PE that receive an initial follow-up* within the first 7 days of discharge.

Denominator 1: The number of patients treated via an outpatient pathway with a new diagnosis of PE. *Initial follow-up can either be via telephone or face to face.

Numerator 2: The number of patients treated via an out-patient pathway for an acute PE that are reviewed within 6 months of diagnosis, subsequent to their initial 7 day review, by a clinician with a special interest in PE as part of a formal pathway ${ }^{\star *}$

Denominator 2: The number of patients treated via an outpatient pathway with a new diagnosis of $\mathrm{PE}^{\star *}$

**Local pathways may define certain groups of patients who do not require this later review (eg, patients with certain forms of malignancy). In such instances, these groups of patients should be excluded from the numerator and denominator. 


\section{Description of what the quality statement means for each audience}

\section{Service providers:}

- Ensure systems are in place such that all patients are managed in line with up to date evidence and guidance.

- Ensure a robust pathway for the outpatient management of patients with PE is in place.

- Will identify a dedicated clinical lead.

Healthcare professionals:

- Ensure all patients are referred into a local PE pathway for ongoing assessment and management. Commissioners:

- Commission local PE outpatient services to ensure all patients have access to follow-up.

People with confirmed new PE:

- Expect robust follow-up.

- BTS Guideline for the Initial Outpatient Management of Pulmonary Embolism (2018). ${ }^{2}$

- NICE Quality Standards 29 (2016). ${ }^{4}$

indicators/data sources

Source references
- BTS Guideline for the Initial Outpatient Management of Pulmonary Embolism (2018). ${ }^{2}$

- Venous thromboembolic diseases: diagnosis, management and thrombophilia testing (2015). ${ }^{3}$

\section{Author affiliations}

${ }^{1}$ Pulmonary Vascular Disease Unit, Sheffield Teaching Hospitals NHS Foundation Trust, Sheffield, UK

${ }^{2}$ Respiratory Medicine, Liverpool University Hospitals NHS Foundation Trust, Liverpool, UK

${ }^{3}$ Haematology, University Hospital of Wales, Cardiff, UK

${ }^{4}$ Thrombosis and Coagulation, King's College Hospital, London, UK

${ }^{5}$ Emergency Medicine, Salford Royal NHS Foundation Trust, Salford, UK

${ }^{6}$ Emergency Medicine, St Thomas' Hospital, London, UK

${ }^{7}$ Royal Free London NHS Foundation Trust, Barnet, UK

${ }^{8}$ Respiratory Medicine, Southampton General Hospital, Southampton, UK

${ }^{9}$ Respiratory Medicine, Papworth Hospital NHS Foundation Trust,

Cambridge, UK

${ }^{10}$ Respiratory Medicine, George Eliot Hospital NHS Trust, Nuneaton, UK

${ }^{11}$ Anticoagulation and Medication Safety/Clinical Governance, Chelsea and

Westminster Hospital NHS Foundation Trust, London, UK

${ }^{12}$ Respiratory Medicine, Kings College Hospital, London, UK

${ }^{13}$ Respiratory Medicine, Royal United Hospital Bath NHS Trust, Bath, UK

Contributors RC was the lead author responsible for the overall editing and production of the document. All authors were responsible for the final approval of the document.

Funding The authors have not declared a specific grant for this research from any funding agency in the public, commercial or not-for-profit sectors.

Competing interests All authors completed a Declarations of Interest form in line with the BTS Policy for Declarations of Interest and forms are available on request from BTS Head Office. RC received funding from Actelion and MSD. PA received funding from Chiesi. RA received funding from Daiichi and from BMS, Pfizer and Bayer. RL received funding from Bayer and Actelion. MN received funding from MSD, GSK and Actelion. JS received funding from MSD, Chiesi, GSK, Teva and Johnson and Johnson. EG, LH, SP, WP, DH, LJS and PJ had no interests to declare.

Patient and public involvement Patients and/or the public were involved in the design, conduct, reporting or dissemination plans of this research. Refer to the 'Methods' section for further details.

Patient consent for publication Not required.

Provenance and peer review Not commissioned; internally peer reviewed.

Open access This is an open access article distributed in accordance with the Creative Commons Attribution Non Commercial (CC BY-NC 4.0) license, which permits others to distribute, remix, adapt, build upon this work noncommercially, and license their derivative works on different terms, provided the original work is properly cited, appropriate credit is given, any changes made indicated, and the use is non-commercial. See: http://creativecommons.org/ licenses/by-nc/4.0/.

\section{REFERENCES}

1 NICE. Quality standards Process guide. London: NICE, 2016.

2 Howard LSGE, Barden S, Condliffe R, et al. British Thoracic Society guideline for the initial outpatient management of pulmonary embolism (PE). Thorax 2018;73.

3 NICE. Venous thromboembolic diseases: diagnosis, management and thrombophilia testing. London: NICE, 2015.

4 NICE. Quality Standard Venous thromboembolism in adults: diagnosis and management. London: NICE, 2016.

5 Standards for Ambulatory Emergency Care. Royal College of physicians of Edinburgh and society of acute medicine, 2019.

6 Aujesky D, Obrosky DS, Stone RA, et al. Derivation and validation of a prognostic model for pulmonary embolism. Am J Respir Crit Care Med 2005;172:1041-6.

7 Jiménez D, Aujesky D, Moores L, et al. Simplification of the pulmonary embolism severity index for prognostication in patients with acute symptomatic pulmonary embolism. Arch Intern Med 2010;170:1383-9.

8 Zondag W, Mos ICM, Creemers-Schild D, et al. Outpatient treatment in patients with acute pulmonary embolism: the Hestia study. $J$ Thromb Haemost 2011;9:1500-7.

9 Otero R, Uresandi F, Jiménez D, et al. Home treatment in pulmonary embolism. Thromb Res 2010;126:e1-5.

10 Aujesky D, Roy P-M, Verschuren F, et al. Outpatient versus inpatient treatment for patients with acute pulmonary embolism: an international, open-label, randomised, non-inferiority trial. Lancet 2011;378:41-8

11 Piran S, Le Gal G, Wells PS, et al. Outpatient treatment of symptomatic pulmonary embolism: a systematic review and metaanalysis. Thromb Res 2013;132:515-9.

12 Davies CWH, Wimperis J, Green ES, et al. Early discharge of patients with pulmonary embolism: a two-phase observational study. Eur Respir J 2007;30:708-14.

13 Agterof MJ, Schutgens REG, Snijder RJ, et al. Out of hospital treatment of acute pulmonary embolism in patients with a low NTproBNP level. J Thromb Haemost 2010;8:1235-41.

14 Vali Y, Ladwa R, Bailie E, et al. Investigating and managing suspected pulmonary embolism in an outpatient setting: the Leicester experience. Thorax 2015;70:291-3.

15 Elf JEJ, J.;Bajc M. Home treatment of patients with small to medium sized acute pulmonary embolism. J Thromb Thrombolysis 2014.

16 Dentali F, Di Micco G, Giorgi Pierfranceschi M, et al. Rate and duration of hospitalization for deep vein thrombosis and pulmonary embolism in real-world clinical practice. Ann Med 2015;47:546-54. 